Supporting Information

\title{
Self-pillared ZSM-5-supported Ni nanoparticles as an efficient catalyst for upgrading oleic acid to aviation-fuel-range-alkanes
}

\author{
Fuxiang Feng ${ }^{a}$, Li Wang $^{a, b^{*}}$, Xiangwen Zhang ${ }^{a, b}$, Qingfa Wang ${ }^{a, b^{*}}$
}

${ }^{a}$ Key Laboratory of Green Chemical Technology of Ministry of Education, School of Chemical Engineering and Technology, Tianjin University, Tianjin 300072, China.

${ }^{\mathrm{b}}$ Collaborative Innovation Center of Chemical Science and Engineering (Tianjin), Tianjin University, Tianjin 300072, China.

* Corresponding author: Tel \& fax: +86 2227892340.

E-mail address: wlytj@tju.edu.cn (L. Wang), qfwang@tju.edu.cn (Q. Wang). 


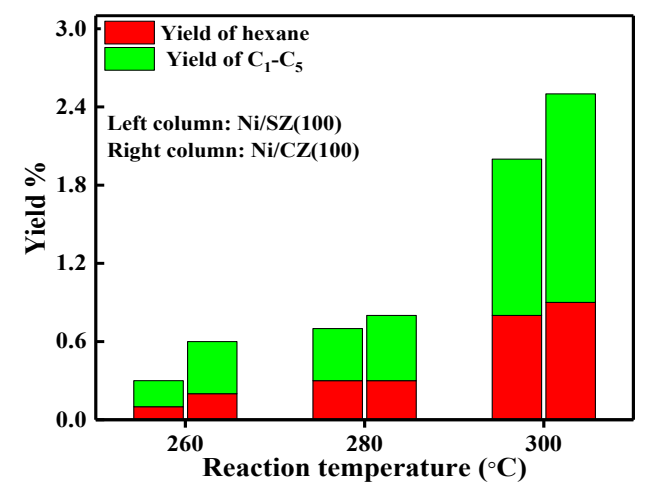

Figure S1. The results for the conversion of cyclohexane over the Ni/SZ(100) and $\mathrm{Ni} / \mathrm{CZ}$ catalysts.

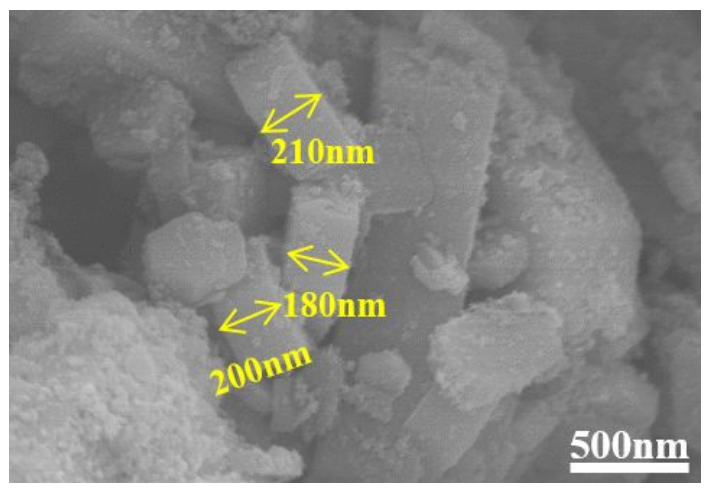

Figure S2. The TEM images of conventional ZSM-5 zeolite.
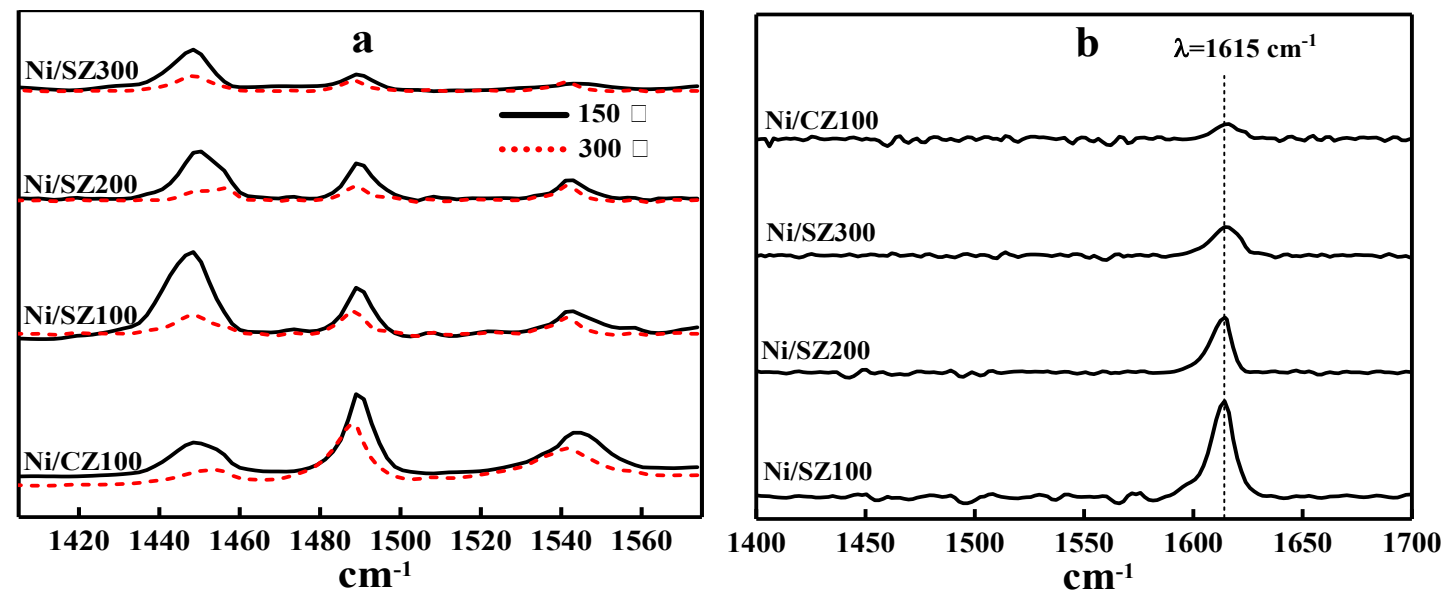

Figure S3. The IR spectra of (a) Py and (b) DTBPy adsorbed on the SP and conventional Ni/ZSM-5 catalysts. 

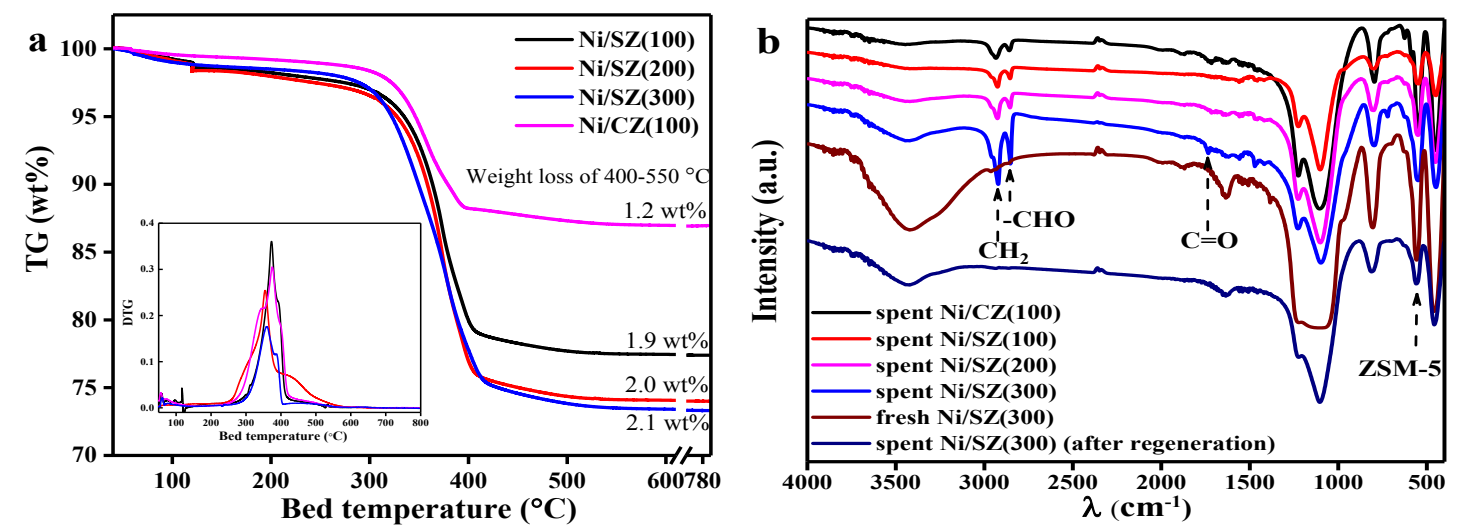

Figure S4. (a) TG curves and (b) FT-IR spectroscopic results for the SP and conventional Ni/ZSM-5 catalysts.

Table S1. Acidity properties of the SP and multilamellar Ni/ZSM-5 catalysts with $\mathrm{Si} / \mathrm{Al}=300$.

\begin{tabular}{ccccccccc}
\hline \multirow{2}{*}{ catalysts } & \multicolumn{2}{c}{ strong $(\mu \mathrm{mol} / \mathrm{g})$} & \multicolumn{2}{c}{ weak $(\mu \mathrm{mol} / \mathrm{g})$} & \multirow{2}{\text{total}}{} & $\mathrm{B} / \mathrm{L}$ & $f_{\text {ext }}$ \\
\cline { 2 - 5 } & $\mathrm{B}(\mathrm{py})$ & $\mathrm{L}(\mathrm{py})$ & $\mathrm{B}(\mathrm{py})$ & $\mathrm{L}(\mathrm{py})$ & $(\mu \mathrm{mol} / \mathrm{g})$ & \\
\hline Multilamellar & 8 & 18 & 3 & 32 & 61 & 0.18 & 0.62 \\
SP & 4 & 14 & 3 & 27 & 48 & 0.17 & 0.40 \\
\hline
\end{tabular}

Table S2. Porous properties of the spent and fresh Ni/ZSM-5 catalysts.

\begin{tabular}{cccccc}
\hline samples & $\begin{array}{c}\mathrm{S}_{\mathrm{BET}} \\
\left(\mathrm{m}^{2} / \mathrm{g}\right)\end{array}$ & $\begin{array}{c}\mathrm{S}_{\text {ext }} \\
\left(\mathrm{m}^{2} / \mathrm{g}\right)\end{array}$ & $\begin{array}{c}\mathrm{S}_{\text {micro }} \\
\left(\mathrm{m}^{2} / \mathrm{g}\right)\end{array}$ & $\begin{array}{c}\mathrm{V}_{\text {meso }} \\
\left(\mathrm{cm}^{3} / \mathrm{g}\right)\end{array}$ & $\begin{array}{c}\mathrm{V}_{\text {micro }} \\
\left(\mathrm{cm}^{3} / \mathrm{g}\right)\end{array}$ \\
\hline $\mathrm{Ni} / \mathrm{SZ}(300)$ & 546 & 300 & 246 & 0.573 & 0.113 \\
Spent Ni/SZ(300) & 432 & 204 & 228 & 0.403 & 0.104 \\
$\mathrm{Ni} / \mathrm{CZ}(100)$ & 245 & 72 & 173 & 0.047 & 0.115 \\
Spent Ni/CZ(100) & 215 & 64 & 151 & 0.040 & 0.100 \\
\hline
\end{tabular}

